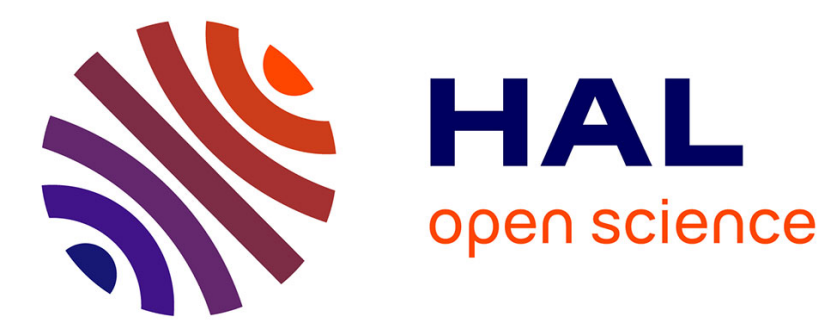

\title{
Equilibrium shape of lyotropic cubic monocrystals
}

P. Sotta

\section{To cite this version:}

P. Sotta. Equilibrium shape of lyotropic cubic monocrystals. Journal de Physique II, 1991, 1 (7), pp.763-772. 10.1051/jp2:1991108 . jpa-00247554

\section{HAL Id: jpa-00247554 https://hal.science/jpa-00247554}

Submitted on 1 Jan 1991

HAL is a multi-disciplinary open access archive for the deposit and dissemination of scientific research documents, whether they are published or not. The documents may come from teaching and research institutions in France or abroad, or from public or private research centers.
L'archive ouverte pluridisciplinaire HAL, est destinée au dépôt et à la diffusion de documents scientifiques de niveau recherche, publiés ou non, émanant des établissements d'enseignement et de recherche français ou étrangers, des laboratoires publics ou privés. 
Classification

Physics Abstracts

$6130-6810$

\title{
Equilibrium shape of lyotropic cubic monocrystals
}

\author{
P. Sotta \\ Laboratoıre de Physıque des Solıdes (*), bâtıment 510, Unıversıté Pans-Sud, 91405 Orsay Cedex, \\ France
}

(Recelved 20 February 1991, accepted 9 Apral 1991)

\begin{abstract}
Résumé. - On décrit le facettage de bulles d'arr incluses dans de très gros monocristaux de phase cubique lyotrope La forme des bulles, qui forment des "cristaux négatıfs", est analysée on montre que les facettes correspondent aux plans réticularres $\{211\}$ du cristal, qui sont précısément les plans de plus grande distance inter-rétıcularre. Il est suggéré que la forme observée est proche d'une forme d'equilibre Des terrasses macroscopıques, de forme polygonale, sont également observées sur les facettes
\end{abstract}

\begin{abstract}
The faceting of air bubbles included in large monocrystals of a lyotropic cubic phase is described The shape of the bubbles, which form «negative crystals », is analyzed; the facets are found to corrrespond to $\{211\}$ reticular planes of the crystal, which are indeed the reticular planes having the largest inter-retucular spacing It is suggested that the observed shape is close to an equilibrium shape Polygonal macroscopic terraces are also observed on the facets
\end{abstract}

\section{Introduction.}

Lyotropic liquid crystals are formed by amphiphilic molecules in the presence of water Amongst them, cubic phases have been extensively studied in recent years [1, 2] These phases are characterized by the presence of a true 3-dimensional crystalline long range order, which gives rise to true Bragg reflections. The crystalline order has been studied mainly by diffraction techniques, namely $X$ ray and neutron scattering experiments Electron microscopy on freeze fractured samples, has also been largely used [3-6]. They are "soft " crystals, with a lattice parameter of the order $100 \AA$ and a unit cell containing typically about $10^{3}$ amphiphilic molecules The molecular organization in these phases consists in a partition of space in two media, one aqueous, one hydrophobic (constituted by the paraffinic tails of the amphiphiles), separated by a surface which is materialized by the polar heads of the amphiphiles The crystalline order onginates in the triply periodic character of this surface [7, 8] Several space groups have been found, and it is not yet entirely clear whether distinct structures having the same space group can exist

A new manifestation of the crystalline order in the cubic phase of the lyotropic system $\mathrm{C}_{12} \mathrm{EO}_{6} /$ Water is described herein We show that «negative crystals", 1.e air bubbles

(*) Associé au CNRS (LA 02) 
included in a monocrystal of cubic phase, exhibit a faceted shape Though the volume of the " negative crystal » 1s fixed by the amount of air trapped withın the bubble, kinetics of surface reorganization play a role in the appearance of facets However, it is argued that the facets which are formed (which are all equivalent) represents the surface equilibrium state, determined by surface tension properties Few systems are known to give rise to crystal equilibrium shape, in a way reliable for quantitatıve experıments Indeed, real shapes result most of the time from growth kinetics anısotropy rather than thermodynamic equilibration [ 9 , 10]

The phase diagram of the binary system $\mathrm{C}_{12} \mathrm{EO}_{6} /$ Water, taken from reference [11], is represented in figure 1 The cubic phase lies in between the lamellar and hexagonal phases, at intermediate temperatures and concentrations. It can be identified easily by observation between cross polarizers due to its isotropy, the phase appears entirely black, without any visible texture We chose this system because its structure has been extensively studied and may be considered to be well established [12] A widespread agreement seems to exist on an organization in two bicontinuous, interwoven amphiphilic labyrinths, separated by an aqueous film which has the topology of the G minimal surface (Schwarz's gyroid) [12-14] These labyrinths are schematized in figure 2. Note that this structure might be representative of several known cubic phases, which space group Ia3d [1]. Moreover, the ability of this system to grow large monocrystals has already been noticed and has allowed a complete structural study, including rather large lattice fluctuations with a well defined geometry [12].

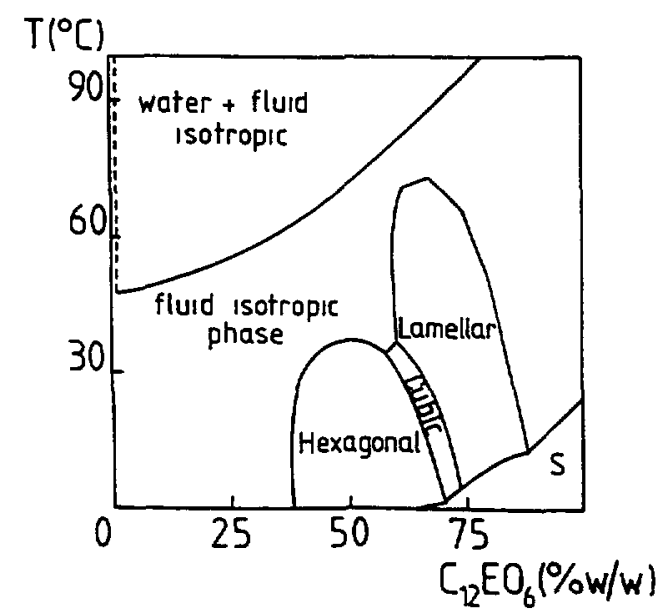

Fig 1 - Phase diagram of $\mathrm{C}_{12} \mathrm{EO}_{6} / \mathrm{H}_{2} \mathrm{O}$, from reference [11] Phase frontiers consist actually in narrow biphasic zones

This is to our knowledge one of the first observations of this kind in lyotropic cubic phases Some authors, however, have observed a facetıng of water drops expelled from a presumably cubic phase upon heating [15] Some other examples are cited in reference [16]. Moreover, crystal shapes have been studied in some other "soft » molecular crystals. Blue phases I and II are cubic phases appearing in cholesteric thermotropic liquid crystals, over a very narrow temperature range They are described as cubic organizations of double-twist cylinders [17] with giant lattice parameter (typically $a=02 \mu \mathrm{m}$ ) and therefore an enormous number of molecules per unit cell (of the order $10^{7}$ ). Facetıng has been observed in Blue Phase I on 


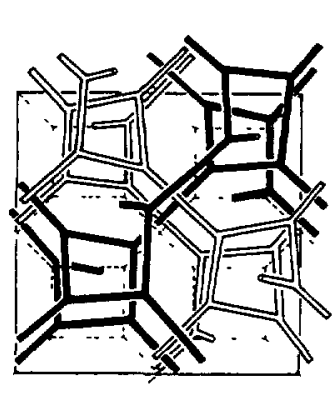

(a)

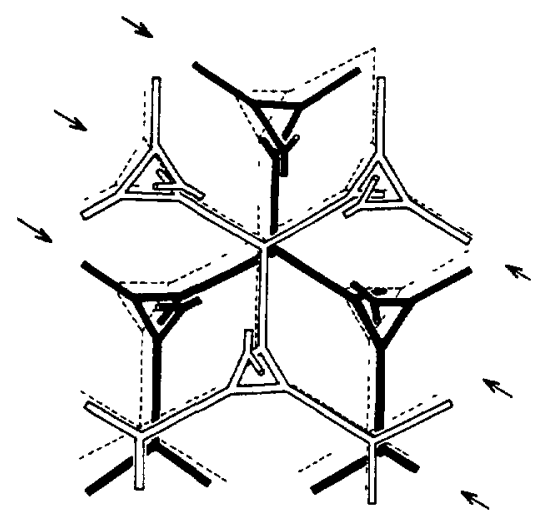

(b)

Fig 2 - The model of structure of a cubic phase with space group la3d, from reference [13] (see also Ref [12]) Black and white rods are the cores of each of the amphiphilic labyrinths a) seen along $\langle 100\rangle$ axis Two cubic cells lie along the vertical $\langle 100\rangle$ axis The square in full line represents the limits of the horizontal face of the unit cell b) seen along $\langle 111\rangle$ axis, which is a 3-fold symmetry axis $\{211\}$ reticular planes are indicated by arrows

crystallites of the order $200 \mu \mathrm{m}$ in size, grown within the surrounding isotropic phase [18-20]. This size represents about $10^{3}$ times the lattice parameter Faceting was also studied in plastic crystals [21-24] wherein true 3D long range order coexists with rotational freedom in each lattice site These crystals are characterized by rather high melting point and fast diffusion of the molecules within the lattice. As in our case, the authors observed faceted equilibrium shapes of bubbles included in crystals

The problem of equilibrum shapes of crystals may be tackled in two ways [10] The first way takes as a phenomenological parameter the surface tension, considered as a function of the surface orientation The surface free energy is to be minimized at equilibrium, under the constraint of constant volume. Facets are then associated with the presence of discontinuities in the derivatives of the surface tension [25] A microscopic approach requires computing the surface tension from a model for the crystalline surface $[26,27]$. In microscopic models, the surface tension is related to the equilibrium distribution of lattice steps on the crystal surface The free energy of a step contains an energy term and an entropy term associated with random wandering of the step on the surface at non-zero temperature This entropy term makes the free energy of a step vanish at a temperature $T_{\mathrm{R}}$ (roughening temperature) given by [28]

$$
k_{\mathrm{B}} T_{\mathrm{R}} \approx \frac{2}{\pi} \gamma d^{2}
$$

where $\gamma$ is the surface stiffness and $d$ is the inter-reticular spacing for the plane corresponding to the surface The crystal shape will then be represented by a distribution of steps that persist at a given temperature $T$ This leads to an equilibrium between flat facets of small Miller indices ( 1 e. large inter-reticular spacings $d$ ) and rounded («rough ») parts [10] Note that direct optical observation of steps was possible in blue phases, due to their giant lattice parameter [19] Roughly speakıng, in accordance with equation (1), the most stable facets, i e those with higher occurrence, often correspond to reticular planes with the largest interreticular spacings 
Apart from faceting, another way to minimize surface energy would be to introduce bulk distortions in the crystal, as far as the interfacial energy is a function of the relative orientation of the surface with respect to the crystal axis. In usual 3D crystals, which are rather "stiff », bulk distortions, such as disclinations, are much more costly than surface terms, and their effect on crystal shapes may be neglected completely However, this is not necessarily the case in « soft " crystals. Indeed, it has been demonstrated recently that the equilibrium shape of a smectic crystal (which is a 1D layered liquid crystal) involves volumic faceting with bulk distorsions of low energy (focal conics), rather than surface faceting [29] In that scope, observing a so well developed faceting in soft crystals is not entirely trivial The 3D structure probably plays an essential role in this phenomenon

Finally, as in usual crystal, structural pieces of information may be drawn from the geometry and specifically the symmetries of the faceted shapes, allowing a kind of macroscopic crystallography in direct space. This information might complement usual powerful diffraction techniques and electronic microscopy Equilibrium shapes may also give an access to the variations of surface tension as a function of surface onentation These variations also would give some structural insight, for example on the density of different reticular planes. However, a knowledge of the local surface structure of the crystal (how do the amphiphilic labyrinths " heal " at the surface ") is important to understand in detals the faceting phenomenon. This question requires additional microscopic experiments, which are quite far from the purely macroscopic observations described here

We first describe the sample preparation and the experimental setup (Sect 2) Then we display the observations and propose a geometrical model for the polyhedral shape observed (Sect 3) This model is constructed with $\{211\}$ reticular planes, it is fully coherent with previous structural studies in this system. Particularly, the specific role played by $\{211\}$ reticular planes is discussed (Sect 4). In section 5, we discuss some irregularities which appear in facet surfaces These consist in polygonal steps and terraces of a macroscopic height A conjectural interpretation may be given, in analogy with «Grandjean terraces » in smectic systems.

\section{Experimental.}

The hexa-ethyleneglycol mono n-dodecyl ether $\mathrm{CH}_{3}-\left(\mathrm{CH}_{2}\right)_{11}-\left(\mathrm{O}-\mathrm{CH}_{2}-\mathrm{CH}_{2}\right)_{6}-\mathrm{OH}$ or $\mathrm{C}_{12} \mathrm{EO}_{6}$, a non-ionic surfactant, was purchased from NIKKO Chemicals Co., Ltd, and used without further purification The samples were prepared by weighing the required amounts of surfactant and heavy water in cylindrical Pyrex glass tubes (diameter $16 \mathrm{~mm}$ ), to give a total weight of about $8 \mathrm{~g}$ The uncertainty on concentration $\Delta c / c$ is 0.2 to $05 \%$ Closed tubes were shaken by hand and then heated to about 45 to $50{ }^{\circ} \mathrm{C}$ for 5 days Samples are lamellar at this temperature, which allows rapid mixing of the components. Samples were then taken back to the cubic phase, at room temperature The lamellar-cubic and cubic-hexagonal transition temperatures were checked to be in concordance with that given in the phase diagram, figure 1 , according to the concentration of the particular sample At this stage, air bubbles are often trapped in the samples, either adsorbed on the glass wall or included within the bulk phase An alternative method is to blow air bubbles in the lamellar phase with a very thin pipette, before cooling the sample in the cubic phase Samples were left at room temperature for at least 6 weeks to allow equilibration No microscopic check of the structure was made. The presence of the cubic structure is assumed in view of the highly viscous, isotropic appearance of the phase, and of its situation in the phase diagram in figure 1 The faceting of bubbles itself may also be considered as a manifestation of the cubic structure.

Photographs from two samples are presented

- sample A $\left(65 \% \mathrm{C}_{12} \mathrm{EO}_{6} / 35 \% \mathrm{H}_{2} \mathrm{O}\right)$ lies in a biphasic region (hexagonal/cubic 
coexistence) at the temperature of the observations. It presents a set of air bubbles adsorbed on the vial wall, embedded in the cubic phase part;

- sample B $\left(66.25 \% \mathrm{C}_{12} \mathrm{EO}_{6} / 3375 \% \mathrm{H}_{2} \mathrm{O}\right)$ contains large air bubbles embedded withn the cubic phase, which have been introduced with a pipette as mentioned above.

The bubbles are observed at room temperature $\left(20^{\circ} \mathrm{C} \pm 1{ }^{\circ} \mathrm{C}\right)$ using a Nachet stereomicroscope with variable magnification, with white unpolarized light, either transmitted or reflected or with a combination of both. A camera can be adapted on the stereomicroscope. Only bubbles near the upper wall of the tube may be observed.

\section{Observations : faceted shape of bubbles.}

Figure $3 \mathrm{a}$ shows the set of bubbles included in sample $\mathrm{A}$, and some partial views of the field (3b and $3 c)$.

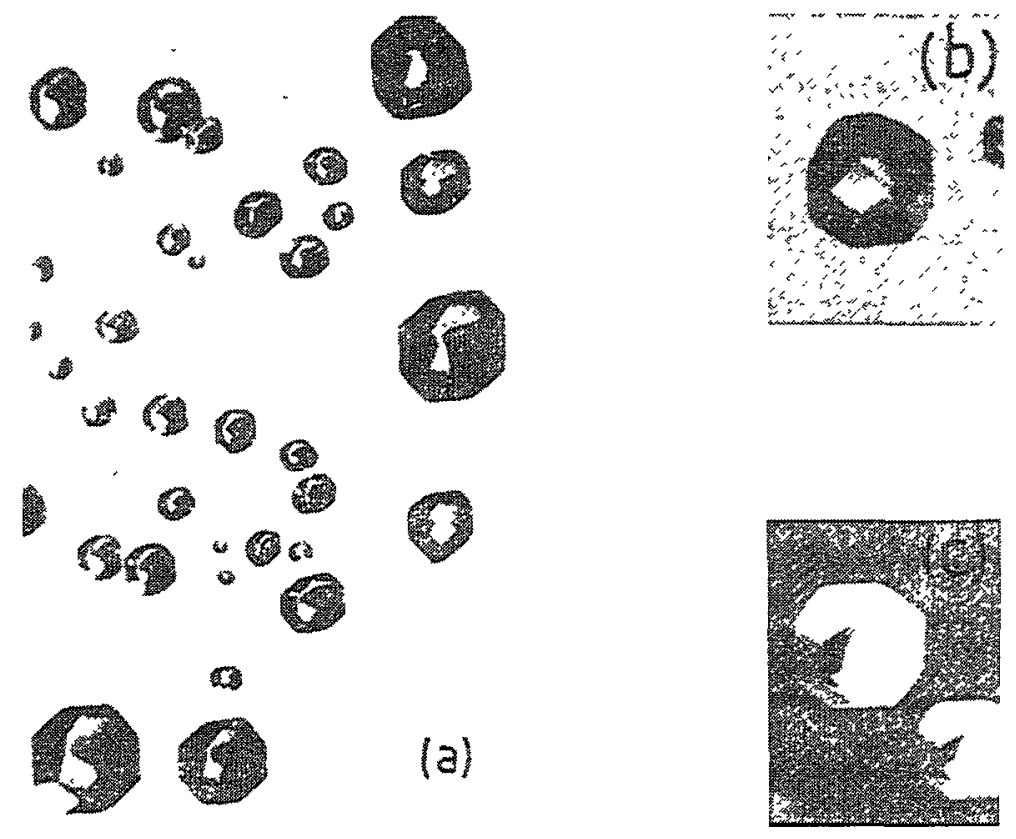

Fig. 3 - Air bubbles included in a cubic monocrystal, adsorbed on the tube wall (sample A). The diameter of the largest one is about $600 \mu \mathrm{m}$. a) The field extends over $6 \mathrm{~mm}$ (transmitted light). b) One of the largest bubble, in the same sample as in (a) (transmitted light). c) Another large bubble (reflected light) A 2-fold axus is almost normal to the honzontal plane. Striations are visible on one facet

A number of bubbles with different sizes exhibit one highly symmetric polyhedral shape. This homothetic invariance strongly suggests that this observed shape corresponds actually to equilibrium, and is not determined by growth or equilibration knetics. There remains some variability in facet extension in some of the bubbles, resulting in an overall distorted habit. However, even in that case, the facet onentations may be clearly identified. Facet onentations for different bubbles are obviously identical, over distances of the order $1 \mathrm{~cm}$. This means that all the corresponding bubbles are embedded within one single crystal. Therefore, this observation indicates that one monocrystal extends over about $1 \mathrm{~cm}^{2}$. 
However, as far as bubbles in this sample lie in the close vicinity of the tube wall, it cannot be asserted that the bulk extension of the monocrystal is larger than the diameter of the larger bubble, 1.e. about $600 \mu \mathrm{m}$. 2-fold axis and 4-fold axis, slightly tilted with respect to the figure plane, may be identified. Intersections between 3 edges are also visible, suggesting the presence of 3-fold axis

A bubble completely embedded in sample $B$ is shown in figure 4 . Its diameter is about $3 \mathrm{~mm}$. It exhibits a perfectly regular polyhedral shape, wherein plane facets coexust with rounded parts. The different pictures illustrate the presence of 2 -fold, 4-fold and 3-fold symmetry axis.

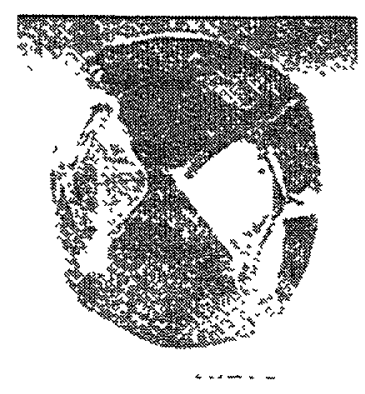

(a)
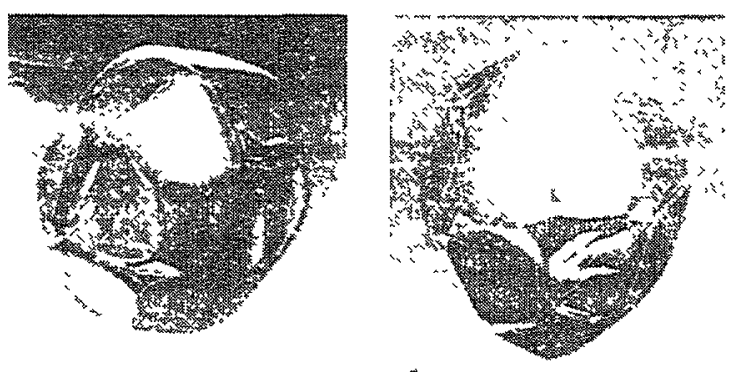

(b)

Fig 4 - Air bubble in sample B (combination of reflected and transmitted light). The overall bubble diameter is about $3.2 \mathrm{~mm}$ : a) seen along a 4-fold axis. b) along a 3-fold axis. c) along a 2-fold axis Terraces are clearly visible The apparent anisotropy (elongation) is due to the conditions of observations, in a cylindncal tube The actual shape is isotropic.

When the sample is cooled down to the hexagonal phase, the bubble shape rapidly alters by flowing. The sample must then be taken back to the cubic phase rapidly. It that conditions, the bubble fully recovers the faceted shape described herein, which is another strong indication for stable equilibnum

As mentioned above, evolution occurs over a time span of a few weeks, after which no further visible (macroscopic) evolution occurs. During this time, the surface reorganizes, either by surface diffusion or local fusion and crystallization via the vapor phase. This evolution is accompanied by a displacement of bubbles towards the top of the crystal (over a distance comparable to bubble diameter). Once the faceted shape is formed, no further displacement is visible. This may be interpreted by a trapping of the facets by the underlying crystalline structure

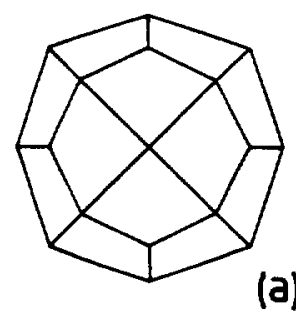

(a)

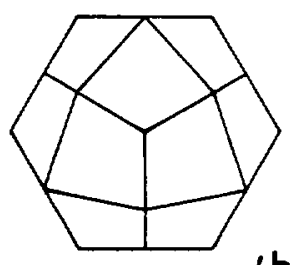

(b)

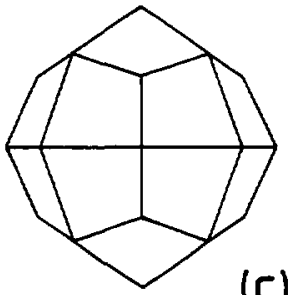

(c)

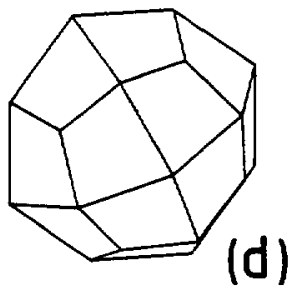

Fig 5. - Polyhedron constructed with $\{211\}$ reticular planes, seen along different axes (a) to (c) are to be compared to figure $4 a$ to $c$ respectively. $d$ is to be compared to figure $3 b$ or $c$. 
The general aspect of the polyhedron, together with the above observations, leads to propose a model constructed with the families of $\{211\}$ reticular planes Figure 5 presents this model polyhedron, projected along different directions The different views in figure 5 are to be compared to those in figures 3 and 4 the concordance between observed and constructed polyhedra is strikıng. Note, for example, that the angle $\alpha$ between 2 adjacent \{211\} planes, as seen on the apparent contour observed along a 4-fold axis is $2 \operatorname{arctg} 1 / 2=53^{\circ}$ This corresponds exactly to the value measured on the bubbles in figure 3

\section{4. $\{211\}$ planes are the most dense reticular planes.}

The model which accounts for the polyhedral shape of the bubbles has been constructed with \{211\} reticular planes The role of these planes in this particular structure is discussed.

The space group has been determined to be Ia3d, by indexing 15 Bragg diffractions [12]. The lattice parameter $a$ found in reference [12] was $118 \AA$ The first, and most intense, Bragg peak precisely corresponds to reflections on $\{211\}$ planes, with an inter-reticular spacing $d=a / \sqrt{6}(=48 \AA$ if one assumes a lattice parameter $a=118 \AA$ ) The absence of the (200) Bragg spot (which is compatible with a bcc structure [30]) is explained by the presence of 4fold screw axis normal to the direction $\langle 100\rangle$ These axis lead to an effective spacing between dense planes along a $\langle 100\rangle$ direction $a / 4$ (1.e. $29.5 \AA$ ), instead of $a / 2(1 \mathrm{e} 59 \AA)$ Similarly, the presence of glide planes perpendicular to $\langle 110\rangle$ leads to a spacing $a / 2 \sqrt{2}$ (1.e. $417 \AA$ ) instead of $a / \sqrt{2}$ ( 1 e. $834 \AA$ ) in this direction

The $\{211\}$ planes are thus the dense planes in the structure with the largest inter-reticular spacing Note that this result does not lie on the model structure pictured in figure 2 , but is infered directly from cristallographic data Moreover, the diffuse scattering analysis in reference [5] indicates that the shear constant from one 3-fold axis to another one is rather weak As a result, shear between $\{211\}$ planes would be relatively easy, since these axis (which are $\langle 111\rangle$ axis) are contained in the $\{211\}$ planes. Additionally, they are the planes which appear most frequently in freeze fracture electron microscopy [5] Note that these planes play also a role in topological transformations involved in phase transitions They are related by epitaxial relations to the planes of the layers in the adjacent lamellar phase, and to the dense planes of the adjacent hexagonal structure [31, 32].

All these points coherently suggest that the $\{211\}$ planes are indeed the planes with the lowest surface energy Therefore the crystal shape constructed with these planes is likely to be close to an equilibrium shape.

Finally, it is worth mentioning that transient shapes seem to appear before the complete equilibrium is attaned These shapes are more faceted than the equilibrium one, and are characterized by the presence of small facets with higher Miller indices, which probably have a higher surface tension and therefore appear as metastable states. $\{220\},\{321\}$ and also small and rare $\{400\}$ facets have been observed These corresponds precisely to the succession of Bragg peaks [5], $1 \mathrm{e}$ to decreasing inter-reticular spacings. Some very small rectangles bounded to certain 2 -fold vertices in figure 3 might be reminiscent of $\{220\}$ metastable facets

\section{Defects in facet surface.}

Remarkable defects on the surface of the facets appear in figure 4, under llluminations at various incidence angles. These consist in polygonal lines, which we interpret as edges of terraces. Obviously these are not constituted by elementary lattice steps their height may be at least measured in microns or tens of microns, which represents $10^{2}$ or $10^{3}$ lattice steps. They seem to be a quite general phenomenon, as they appear also in some facets in figure $3 \mathrm{~b}$ and $\mathrm{c}$ 
under the form of parallel alternatively dark and bright striations The edges have well defined directions in the planes of the facets. The direction occurning most often in each facet (which corresponds also to the direction of the striations in Fig 3) is the diagonal line issuing from the 3-fold vertex Some other steps are parallel to facet edges The very unidirectional striations described above seem to reflect a noticeable anisotropy of surface properties, which may be reflected also in the more pronounced rounding of vertices corresponding to 2-fold axis, than of those associated with 4-fold and 3-fold axis, as is apparent in figure 4c.

The occurrence of these particular directions may be explained as follows The diagonal lines mentioned above correspond to 3 -fold axis $\langle 111\rangle$, contained within the planes of the facets Three $\{211\}$ planes intersect along each of these $\langle 111\rangle$ axis at an angle $60^{\circ}$ (see Fig 2b) Similarly, lines parallel to facet edges correspond to intersections between the given facet plane and the $\{211\}$ planes of adjacent facets [33] So, it appears that the step edges coincide with intersections between $\{211\}$ planes This leads to consider that the step risers consist also in such planes, or, in other words, that one facet plane fluctuates between several $\{211\}$ planes, which all have the same, lowest, surface tension. However, an excess energy is associated with the increase in total area associated with the presence of terraces

Very similar macroscopic terraces ("Grandjean terraces") are observed in layered systems, either thermotropic smectic or lyotropic lamellar phases [34,35] They appear at the free surface of drops deposited on glass slides. In these systems, edge dislocations tend to migrate towards the free surface of the crystal. This attraction, which is a quite general phenomenon, originates from relaxation of bulk elastic stresses Then, due to the specific form of the dislocation cores, which involve mainly curvature of the layers, the energy of a dislocation exhibits a pecular dependence in the core size $\xi$, favoring large Burgers vectors. The dislocations close to the surface then group, which results in macroscopic steps. A similar process might be envisaged in this system, in which $\{211\}$ planes are reminiscent of lamellae planes [31] By analogy, it might be assumed that edge dislocations, attracted to the free surface, tend to group and form «giant» dislocations, which then result in macroscopic steps The additional point to notice here, is that deformations in the vicinity of the surface may be entirely relaxed this is achieved by reorganizing the dislocation cores so that step nisers coincide with $\{211\}$ planes, different from the initial facet plane This process is illustrated in figure 6
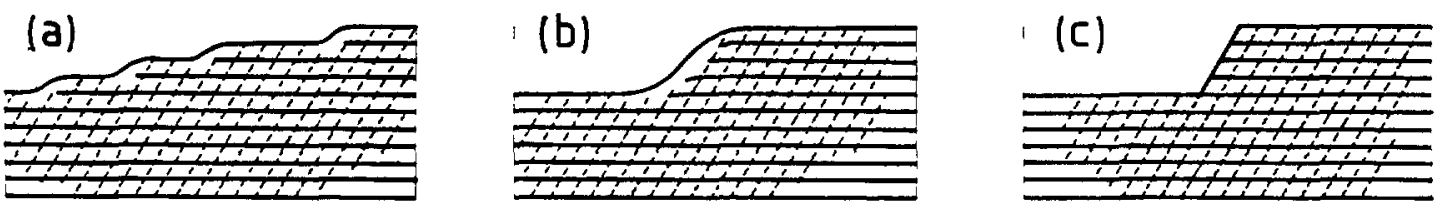

F1g 6 - Relaxation of bulk deformations associated to dislocations near the surface a) Edge dislocations attracted at the free surface of the crystal The layers represent one set of $\{211\}$ planes. Dotted lines are the traces of another set of $\{211\}$ planes, intersecting with that of the layers along a $3-$ fold axis (normal to the plane of the figure) b) Regrouping of dislocations at the surface c) Creation of a $\{211\}$ stripe at the surface Distorsions have been completely relaxed within the bulk crystal

These terraces are probably trapped by the cubic structure in a way similar to the trapping of facets the motion of terrace edges, once formed, involves energy barriers associated to global displacement of $\{211\}$ risers. If they were truly equllibrium patterns, the extra surface energy, associated with the increase in total area, should be at most comparable to bulk dislocation energy [36]. It is interesting to estimate the surface energy $\alpha$, using the above 
criterium Suppose a dislocation (of unit length, and Burgers vector $d$, the inter-reticular spacing of $\{211\}$ planes) is attracted to the surface Its energy may be evaluated in analogy, either with « hard " crystals, or with layered systems. In the first case, it is of the order a few $d^{2} B$, where $B$ is the compressibility modulus perpendicular to $\{211\}$ planes. In the second case, it compares to $d\left(B K_{1}\right)^{1 / 2}$, where $K_{1}$ is a curvature modulus for $\{211\}$ planes, assimilated in that case to the layers In both cases, this is of the order at least a few $10^{-6} \mathrm{erg} \mathrm{cm}^{-1}$, assuming $B \sim 5 \times 10^{6}$ dyne $\mathrm{cm}^{-2}, K_{1} \sim$ a few $10^{-6}$ dyne and $d=50 \AA$ (see Refs. [34] and [35]). When the dislocation is replaced by a $\{211\}$ stripe, the increase in surface energy compares to $\mathrm{d} \alpha$, which should then be of a few $10^{-6} \mathrm{erg} \mathrm{cm}^{-1}$. Such an estimate gives a capillary length $\ell_{c}=(2 \gamma / \rho g)^{1 / 2}$ of the order 1 or $2 \mathrm{~mm}$, which is fairly coherent with the observation of such large nearly spherical bubbles.

\section{Conclusion.}

We have observed the facetıng of «negatıve crystals » included within a lyotropic cubic phase This demonstrates, first, the possibility to obtain very large bulk monocrystals (of the order $10 \mathrm{~mm}^{3}$ to, possibly, $1 \mathrm{~cm}^{3}$ ) Then, we have analyzed the shape of the inclusion as being constructed with $\{211\}$ reticular planes, which are the planes with the largest inter-reticular spacing and the planes of easy fracture In that point of view, lyotropic cubic phases behave in a way very similar to atomic « hard» crystals, wherein dense planes are precisely those with the largest inter-reticular spacing and, correlatively, those of easy fracture and of lowest surface energy.

Finally, it may be noticed that surface faceting prevalls over bulk distorsions in this crystal, contrary to bulk lamellar systems Indeed, in 1D structures, structural displacements of large amplitude (disclinaisons) are possible. This allows bulk distorsions with an energy scaling linearly with the size of the system [29] In a 3D structure, even though it is a quite "soft" crystal, these are probably by far too much costly

\section{Acknowledgments.}

I am particularly indebted to J. Charvolin who introduced me in the fascinating word of cubic phases J B Foumier gave me a preprint of reference 29, prior to publication. Thanks are also due to the referees for their critics and comments

\section{References}

[1] An extensive review is given in Fontell K, Colloid Polym Scl 268 (1990) 264

[2] Charvolin J, J Phys. Colloq. France 46 (1985) C3

[3] Verleij A J, Blochim Biophys Acta 779 (1984) 43

[4] Lindblom G, Rilfors L, Blochm Blophys Acta 998 (1989) 221

[5] Delacroix H, Mariani P, Gulik-Krzywicki T, J Phys. Collog France 2351 (1990) C7-119

[6] Gulik-KRZYWICKI T, AGgerbeCK L P, LARSSON K, in « Surfactants in Solution ». K L. Mittal \& B Lindman Eds (Plenum Press) 1983

[7] Sadoc J F. Charvolin J, $J$ Phy:s France 47 (1986) 683

[8] Charvoliv J, Contemporary Phy's 31 (1990) 1

[9] Balibar S, Gallet F, Graner F, Rolley E, Physica A 163 (1990) 111

[10] WORTIS M, in "Chemistry and Physics of Solid Surfaces", R Vanselow Ed (Springer Verlag, Berlin) 1988, Vol VII

[11] Mitchell D J, Tiddy G. J T, Waring L, Bostock T, McDonald M P, $J$ Chem Soc Faraday Trans 79 (1983) 975 
[12] Rançon Y, Charvolin J, J Phys France 48 (1987) 1067.

[13] LuZZATI V, SPEGT P A, Nature 215 (1967) 701

[14] Luzzati V , Tardieu A, Gulik-Krzywicki T , Rivas E, Reiss-Husson F , Nature 220 (1968) 485

[15] Tiddy G J T, Rendall K, Galsworthy P, Mol Cryst Llq Cryst Lett. 72 (1982) 147

[16] Winsor P. A, in «Liquid Crystals and Plastic Crystals», Vol 1, G W Gray \& P A Winsor Eds, (Ellis Harwood Ltd, Chichester) 1974

[17] Dubois-Violette E., Pansu B., Mol Cryst Liq Cryst 165 (1988) 151

[18] Barbet-Massin R., Cladis P E, Pieranski P, Phys. Rev A 30 (1984) 1161

[19] Pieranski P, Barbet-Massin R, Cladis P E, Phys. Rev A 31 (1985) 3912

[20] Pieranski P, Cladis P E, Barbet-Massin R, J Phys France 47 (1986) 129

[21] Pavlovska A, Nenow D, Surface Scr. 27 (1971) 211.

[22] Pavlovska A, Nenow D., J Cryst Growth 8 (1971) 209

[23] Pavlovska A, Nenow D., J. Cryst Growth 12 (1972) 9.

[24] Pavlovska A, Nenow D, J. Cryst Growth 39 (1977) 346

[25] Landau L, Lifshitz E, Stat Phys (MIR, Moscov).

[26] Rottman C, Wortis M, Phys. Rep. 103 (1984) 59.

[27] Schulz H. J, J. Phys France 46 (1985) 257.

[28] Fisher D S, WeEks J D, Phys. Rev Lett 50 (1983) 1077

[29] Fournier J B, DURAND $G$, to be published

[30] All Bragg spots $(h k \ell)$ with $h+k+\ell$ odd are forbidden by the bcc symmetry, in particular, this means that the effective spacing between dense planes along a $\langle 111\rangle$ direction is $a / 2 \sqrt{3}$ (1 e $34 \AA$ ) instead of $a / \sqrt{3}$, and $a / 2 \sqrt{5}$ (1.e $264 \AA$ ) instead of $a / \sqrt{5}$ along a $\langle 210\rangle$ direction

[31] RançON Y, Charvolin J., $J$ Phys Chem 92 (1988) 2646

[32] Rançon Y, Charvolin J., J. Phys Chem 92 (1988) 6339

[33] Intersections of a facet with the planes of non-adjacent facets would correspond to other lines in its plane In this case, the angles between intersecting planes are larger, closer to $90^{\circ}$ The extra energy associated with edges (or furrows) may be higher in that case

[34] De Gennes P G, «The Physics of Liquid Crystals » (Clarendon Press, Oxford) 1974.

[35] KLÉmAN M , "Points, Lines and Walls » (John Wiley \& Sons) 1983

[36] There remains also a contribution from edges (or furrows), which should be comparable to the energy associated to " unit» dislocation cores 